\title{
DETECTION OF STREET LIGHT POLES IN ROAD SCENES FROM MOBILE LIDAR MAPPING DATA FOR ITS APPLICATIONS
}

\author{
Siamak Talebi Nahr ${ }^{\text {a, } * \text {, Mohammad Saadatseresht }}{ }^{\text {a }}$ Jamshid Talebi $^{\text {b }}$ \\ ${ }^{a}$ School of Surveying and Geospatial Engineering, College of Engineering, University of Tehran \\ b Pooya-Naghsh-Omid Consulting Engineers Company, 021-44095520, pooya.naghsh@gmail.com
}

KEY WORDS: MLS, light pole, Bhattacharya, SMRF, DBSCAN, ITS

\begin{abstract}
:
Identification of street light poles is very significant and crucial for intelligent transportation systems. Automatic detection and extraction of street light poles are a challenging task in road scenes. This is mainly because of complex road scenes. Nowadays mobile laser scanners have been used to acquire three-dimensional geospatial data of roadways over a large area at a normal driving speed. With respect to the high density of such data, new and beneficial algorithms are needed to extract objects from these data. In this article, our proposed algorithm for extraction of street light poles consists of five main steps: 1. Preprocessing, 2. Ground removal, 3. 3D connected components analysis, 4. Local geometric feature generation, 5. Extraction of street light poles using Bhattacharya distance metric. The proposed algorithm is tested on two rural roadways, called Area1 and Area2. Evaluation results for Area1 report 0.80, 0.72 and 0.62 for completeness, correctness and quality, respectively.
\end{abstract}

\section{INTRODUCTION}

Nowadays, high-density and high-accuracy Mobile Laser Scanning (MLS) data are becoming a primary source for highway mapping (Gong, Zhou et al. 2012), urban road distress assessment (Awrangjeb and Fraser 2014, Guan, Li et al. 2015), and road feature inventory ( $\mathrm{Pu}$, Rutzinger et al. 2011, Guan, Yu et al. 2015). Road feature inventory is a crucial necessity in intelligent transportation systems (ITS) applications. Pole-like road objects, including light poles, telecommunication poles and traffic signposts, located along roads/streets, are typical kinds of road infrastructure. For example, light poles provide illumination to pedestrians and vehicles at night for a clear visibility of the road environment. Traffic signposts, as a highly important transportation infrastructure, play a critical role in ITS, traffic safety, and route guidance. For ITS related applications, the information of pole-like objects can be used for road infrastructure maintenance, road safety analyses, advanced driver assistance, semantic mapping, and smart city applications. For instance, for a driver assistance system, the position of street light poles can be used to improve the stability of road tracking (Fleischer and Nagel 2001). Therefore, regular inventory and maintenance of street light poles is important. Due to the large number of street light poles on road, traditional manual survey methods are extremely time-consuming. A rapid and robust method is highly needed to obtain street light poles information.

Identification of street light poles is very significant and will make the detection of the attached objects easier. Regularly rounded and long, poles are made of different materials and they have different heights and radius. However, in the same scene, the type of poles is usually identical. Thus, recently, detecting street light poles has attracted increased attention in the literature.

MLS systems integrate laser scanner(s), a global navigation satellite system, an inertial measurement unit, a distance measurement indicator, and digital/video camera(s) (Murray, Haughey et al. 2011, Brogan, McLoughlin et al. 2013). MLS systems have been used to acquire three-dimensional (3-D) geospatial data of roadways over a large area at a normal driving speed (Figure 1).

The average density of the point clouds collected by a MLS system can reach up to 4000 points/m2 with a moving speed of approximately $50 \mathrm{~km} / \mathrm{h}$. Therefore, MLS systems provide a promising way to extract street lighting poles. In fact, automated extraction of street lighting poles from MLS point clouds has been an active research topic in recent years.

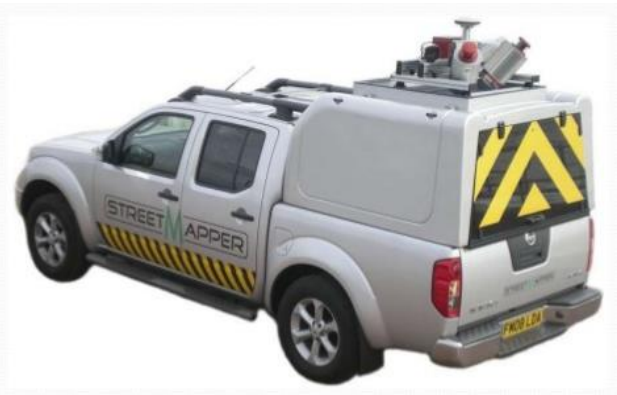

Figure 1. Street Mapper, the LiDAR mobile mapping system utilized in this research.

* talebi@ut.ac.ir 
In this paper, we will propose a procedure to detect street light poles from rural road scenes. Our procedure consists of five main steps: 1. Pre-processing, 2. Ground removal, 3. 3D connected components analysis, 4. Local geometric feature generation, 5. Extraction of street light poles.

\section{RELATED WORKS}

There are many articles for road object detection via MLS data. For example, based on eigenvalue analysis, principal component analysis (PCA) was a widely used method for detecting pole-like road objects from irregular point clouds(Yokoyama, Date et al. 2011, Yokoyama, Date et al. 2013). These methods detected linear pole-like structures by first constructing a covariance matrix for each point with its neighbours and then analysing eigenvalues decomposed from the covariance matrix (El-Halawany and Lichti 2011). Eigenvalue-based PCA methods show high computational efficiency. However, other objects (particularly tree trunks) in a road scene might cause a considerable number of false alarms.

With the prior knowledge of pole-like objects in shape and size, by using grammar rules, a voxel structure was applied to mobile LiDAR data (Cabo, Ordoñez et al. 2014). Through a 3-D neighbourhood analysis of voxel representations, pole-like objects were detected. In (Chen, Zhao et al. 2007, Kukko, Jaakkola et al. 2009, Lehtomäki, Jaakkola et al. 2010, Hu, Li et al. 2011), pole-like objects were extracted by analysing scan lines, rather than raw point clouds. To improve computational efficiency, some studies convert 3-D point clouds into 2-D representations (El-Halawany and Lichti 2013). Point density in 2-D representations was also exploited to detect light poles (Chen, Zhao et al. 2007).

\section{PROPOSED ALGORITHM}

In this section, the proposed algorithm will be discussed. Therefore, in section 3.1 some pre-processing steps to prepare input data for the proposed algorithm are explained. Section 3.2 gives a brief explanation of ground removal algorithm. In section 3.3 a $3 \mathrm{D}$ connected components algorithm is used to find connected objects in Euclidian distance metric. In the following section, local geometric features are generated, and finally in section 3.5 Bhattacharya distance is used to detect street light poles.

\subsection{Pre-processing}

This section intends to reduce huge amount of MLS data. To do this, a buffer is set and the points out of it are eliminated. The centre of buffer is trajectory of sensor and its width is equal to two times of the road width. The reason we do this is because we know street light poles are placed in this range. After eliminating the points out of the buffer, the amount of data will reduce tangibly and the following parts of algorithm will run faster.

\subsection{Ground Removal}

After pre-processing section, the amount of data will be reduced, but because of the high density of MLS data, it is still high for computational purposes. To overcome this problem, a ground removal algorithm is used in this paper.

To remove ground points a Simple Morphological Removing Filter (SMRF) is used in this paper (Pingel, Clarke et al. 2013). The algorithm contains four theoretically distinct phases. The first is the creation of the minimum surface. The second is the processing of the minimum surface, in which grid cells from the raster are identified as either containing bare earth or objects. This second stage signifies the heart of the algorithm. The third step is the creation of a DEM from these gridded points. The fourth step is the identification of the original MLS points as either bare earth or objects based on their relationship to the interpolated DEM.

\subsection{D Connected Components Analysis}

After eliminating ground points, we need to group remaining points into detached objects based on Euclidian distance. On this basis, a 3D connected component extraction algorithm, which is called DBSCAN, is used (Tran, Drab et al. 2013). This algorithm is based on the number of points in a specified distance. The overall steps of this algorithm are shown in Figure 2 .

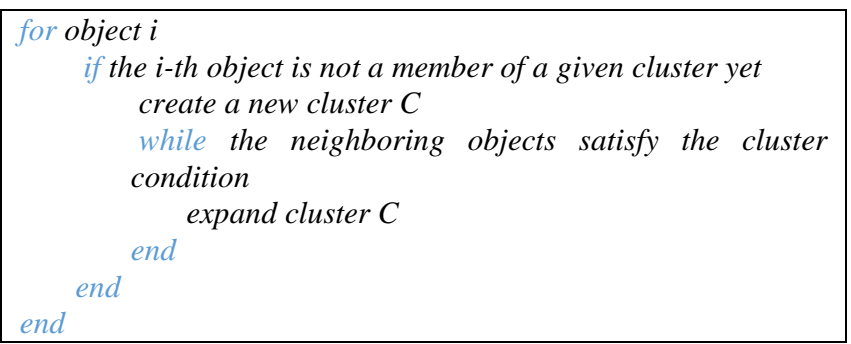

Figure 1. Overall steps of DBSCAN algorithm (Lehtomäki, Jaakkola et al. 2010).

At the end, some previously known properties of street light poles are used to filter unwanted objects. At the following steps, each of these connected objects will be processed separately.

\subsection{Local Geometric Features Generation}

In this paper statistical analysis of local geometric properties are used to generate street light poles features, as they are used in (Zai, Chen et al. 2015). On this basis, for each point of objects a $5 \mathrm{D}$ feature vector is calculated, which represents local geometric features for a point in a specified neighborhood. Equation (1) shows this feature vector:

$F_{p}=\left\{N_{x}, N_{y}, N_{z}\right.$, dis,$\left.n_{\text {var }}\right\}$

Where $N_{x}, N_{y}$ and $N_{z}$ are components of normal vector at a specified neighborhood of point $p$. dis is distance between $p$ and center of gravity of all object points in XY plane. $n_{\text {var }}$ is local variance of normal vectors at a specified neighborhood of point $p$.

After generating $F_{p}$ for all points of an object, in the following step a 5D Gaussian pdf is estimated. If $\Phi_{i}=N_{i}\left(\mu_{i}, \Sigma_{i}\right)$ is 5D Gaussian function for object $i, \mu_{i}$ and $\sum_{i}$ are shown as:

$\mu_{i}=\frac{1}{n} \sum_{j=1}^{n} f_{p j}$

$\sum_{i}=\frac{1}{n} \sum_{j=1}^{n}\left(f_{p j-} \mu_{i}\right)^{T}\left(f_{p j-} \mu_{i}\right)$

\subsection{Extraction of street light poles}

3D connected components usually have similar geometric features. On this basis, probability density functions, which are estimated using these geometric features, should be also similar. 
In this paper Bhattacharya distance is used to discriminate $5 \mathrm{D}$ Gaussian pdfs (Zai, Chen et al. 2015). This metric is computationally simple and can calculate distance between two n-dimensional normal Gaussian pdfs. If $N_{p}=\left\{\mu_{p}, \Sigma_{p}\right\}$ is the Gaussian pdf of a street light pole, its distance to another object's Gaussian pdf, $N_{i}=\left\{\mu_{i}, \sum_{i}\right\}$, could be computed as following equation:

$d_{\text {bhati }}=\frac{1}{8}\left(\mu_{i}-\mu_{p}\right)^{T}\left[\frac{\sum_{p}+\sum_{i}}{2}\right]^{-1}\left(\mu_{i}-\mu_{p}\right)+\frac{1}{2} \ln \frac{\left|\frac{\sum_{p}+\sum_{i}}{2}\right|}{\sqrt{\left|\sum_{p}\right|\left|\sum_{i}\right|}}$

Equation (4) shows the difference between two normal pdfs. The range for Bhattacharya distance is $[0,+\infty]$, in which, 0 means two normal pdfs are identical. Using this metric and a specified threshold similar objects can be extracted.

\section{EXPERIMENTS}

The algorithm was tested using a MLS dataset from the Riegl VMX-250. This system integrates two LIDAR sensors (VQ250), and an IMU/GNSS unit, and it is deployed on the rear top of a van. The VQ-250 is a rotational sensor that acquires points with a $360^{\circ}$ field of view on planes set, in this case, at $45^{\circ}$ to the horizontal and $45^{\circ}$ to the trajectory (i.e. driving direction).

The measurements were made along two rural roads in the north of Iran, called Area1 and Area2. Each of these roads are approximately $350 \mathrm{~m}$ long. Area 1 is placed at the out of the village and Area2 is a road in inside of the village. Therefore, Area2 is a little bit more complex than Area1. These roads have many objects such as bushes, lights, communication poles and trees. To implement proposed algorithm, a buffer with $25 \mathrm{~m}$ width is used to filter unwanted points and decline the huge amount of MLS data. The Figure (3) shows two roads after applying buffer.

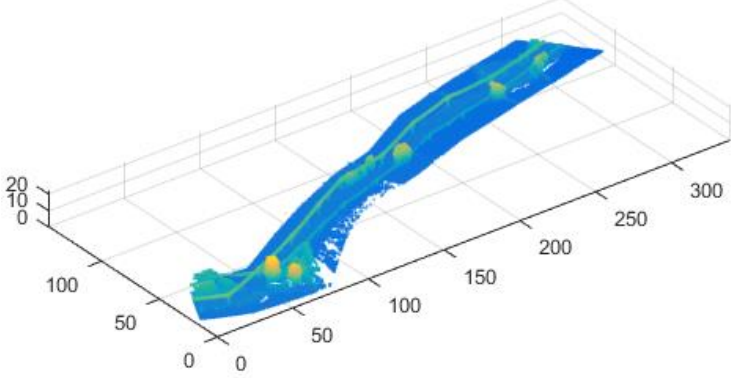

Area1

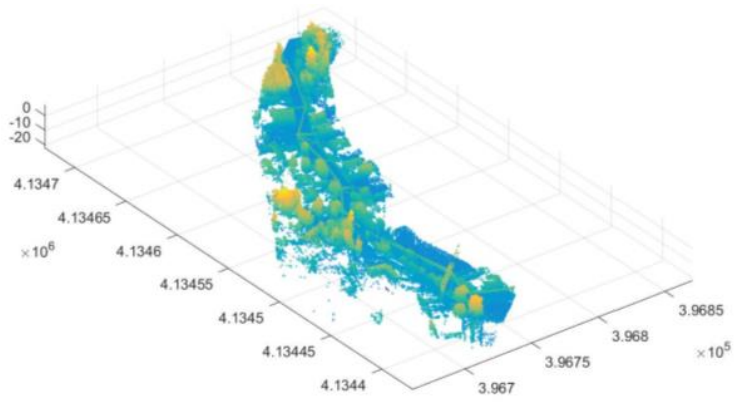

Area2
Although filtering unwanted points declines the amount of data, but it is still a high value and need to be reduced. This is because of high density of MLS points. To do this ground points are removed by SMRF algorithm. The SMRF parameters are shown in Table (1) and non-ground points are shown in Figure 4.

To generate 3D connected components DBSCAN algorithm is used. In this algorithm, search radius and minimum number of points in neighborhood are set to $0.30 \mathrm{~cm}$ and 3 , respectively. Generated connected components are shown in Figure (5).

\section{Cell size $1 \mathrm{~m}$}

Maximum expected slope $\quad 0.2$

Maximum window radius 16

Elevation threshold 0.45

Elevation scaling factor 1.2

Table 1. SMRF algorithm parameters.

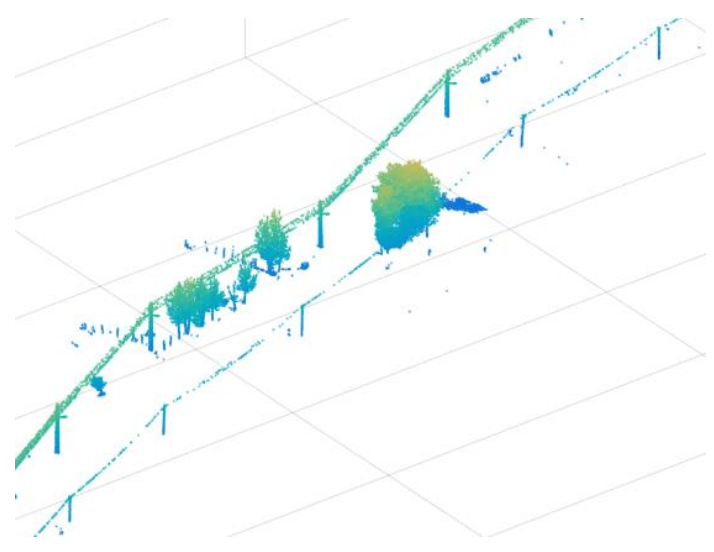

Area1

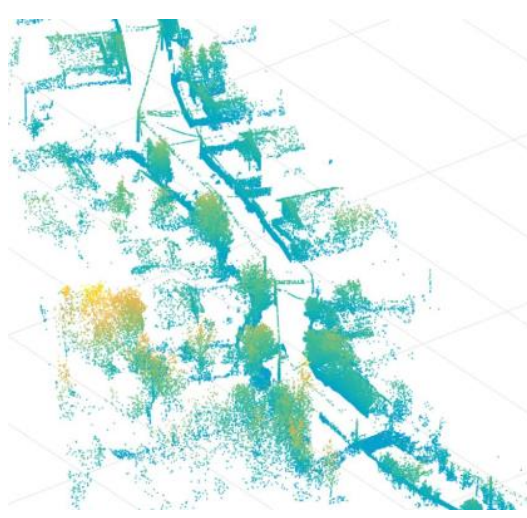

Area2

Figure 4. Non-ground points generated using SMRF.

Number of connected components are 2975 and 3224 objects for Area1 and Area2, respectively. With respect to the primary properties of street light poles, we can filter these connected components to eliminate unwanted objects. On this basis, following criteria are used to filter out unwanted objects:

a. The number of points in each objects should be $[100$, 20000].

b. The height of the objects need to be more than 3 meters.

Figure 3. Test area before and after filtering using $25 \mathrm{~m}$ buffer. 
c. The maximum $\mathrm{Z}$ value of the objects need to be more than 6 meters.

These criteria conclude in 44 and 45 street light pole candidates for Area1 and Area2, respectively.

To generate feature vector (Eq. (1)) 8 neighbors of each point in an object are considered, then a 5D Gaussian pdf is estimated for each object. Next, one of the street light poles is chose manually. At last, Bhattacharya distance between desired light pole and other objects are calculated. Considering 0.4 as a threshold value, all of light poles are extracted. These objects are represented in red color in Figure (6).

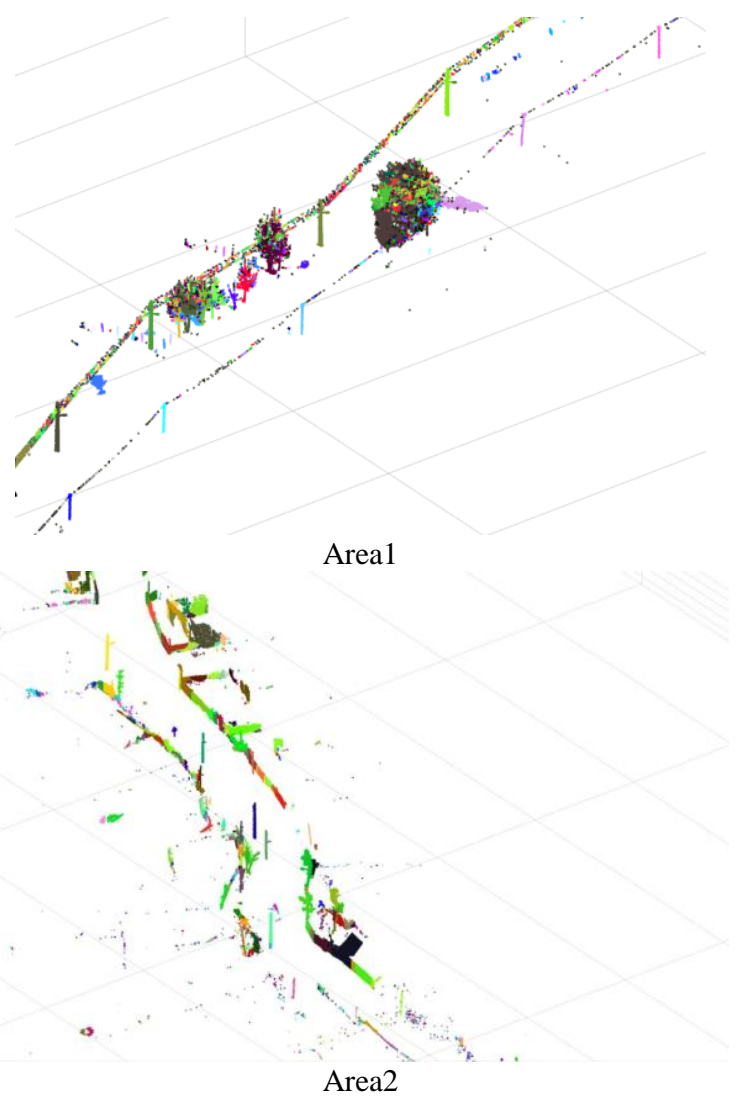

Figure 5. 3D connected components detected using DBSCAN.

\section{EVALUATION}

For evaluation purpose of results, we use completeness, correctness and quality indices presented in (Rutzinger, Rottensteiner et al. 2009). Equations 5 to 7 represent these indices. To analyze threshold impact on the results, evaluation indices are calculated for $0.3,0.4,0.5$ and 0.6 values. Table (2) shows these results.

$$
\begin{aligned}
& \text { Comp }=\frac{T P}{T P+F N} \\
& \text { Corr }=\frac{T P}{T P+F P} \\
& \text { Corr }=\frac{T P}{T P+F P+F N}
\end{aligned}
$$

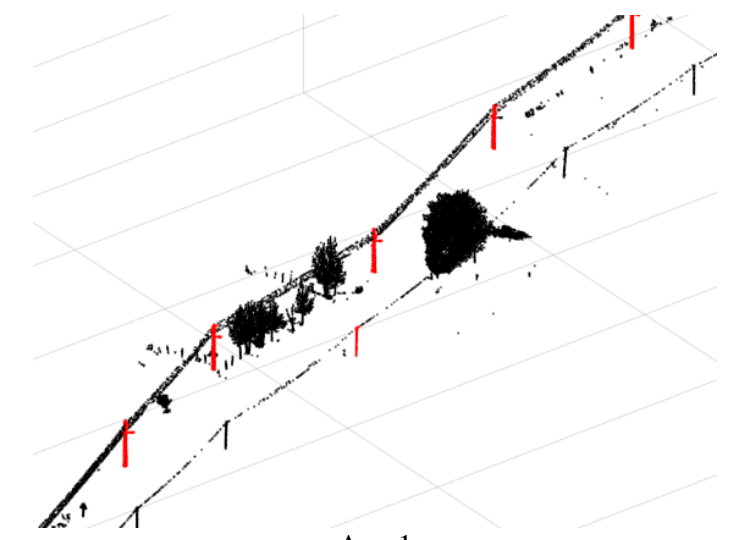

Area1

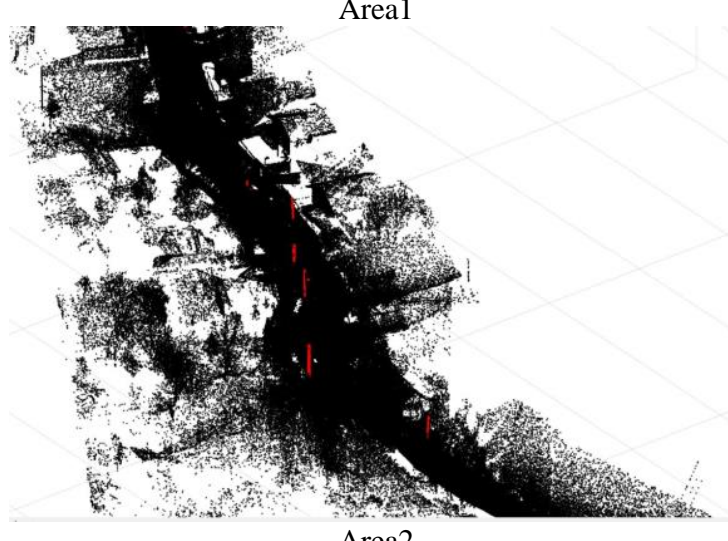

Figure 6. Final results of light poles extraction.

\section{CONCLUSION}

For evaluation purpose of proposed method, we used MLS data of two rural road with length of $350 \mathrm{~m}$ for each of them. There are many entities at the scene such as light poles, trees, telecommunication poles, bushes etc. To extract street light poles firstly SMRF algorithm is used to eliminate ground points. Next using DBSCAN algorithm 3D connected components are detected. Because of huge number of $3 \mathrm{D}$ connected components and with respect to the primary information of street light poles, these components are filtered out to get candidate street light poles. Local geometry features are generated at the next step. After estimating 5D Gaussian pdf on local geometry features, street light poles are extracted using Bhattacharya distance metric.

Completeness, correctness and quality indices are used to evaluate street light poles extraction results. As we can see in Table (2) proposed algorithm reached $0.80,0.72$ and 0.62 for Area1 and $0.79,0.92$ and 0.73 for Area2 as completeness, correctness and quality indices, respectively. These results represent a good performance of proposed algorithm for extraction of street light poles from MLS data in a rural road scene.

At last, considering some flaws and weaknesses of proposed algorithm, such as covered street light poles with trees, it seems using some segmentation algorithms after 3D connected components analysis could help improve results. Besides, manipulating local geometry features vector and using some other feature might be helpful. Finally determining Bhattacharya distance threshold is also a challenging task and could be considered in future researches. 
Area1

\begin{tabular}{ccccccccc}
\hline & \multicolumn{2}{c}{ Ground Truth } & \multicolumn{3}{c}{ Extracted Results } & \multicolumn{3}{c}{ Evaluation Results } \\
Threshold & $\begin{array}{c}\text { Light- } \\
\text { poles }\end{array}$ & $\begin{array}{c}\text { Other } \\
\text { poles }\end{array}$ & TP & FN & FP & Completeness & Correctness & Quality \\
$\mathbf{0 . 3}$ & 10 & 12 & 6 & 4 & 0 & 0.60 & 1.00 & 0.60 \\
$\mathbf{0 . 4}$ & 10 & 12 & 7 & 3 & 1 & 0.70 & 0.88 & 0.63 \\
$\mathbf{0 . 5}$ & 10 & 12 & 8 & 2 & 3 & 0.80 & 0.72 & 0.62 \\
$\mathbf{0 . 6}$ & 10 & 12 & 8 & 2 & 6 & 0.80 & 0.57 & 0.50
\end{tabular}

Area2

\begin{tabular}{ccccccccc}
\hline & \multicolumn{2}{c}{ Ground Truth } & \multicolumn{3}{c}{ Extracted Results } & \multicolumn{3}{c}{ Evaluation Results } \\
Threshold & $\begin{array}{c}\text { Light- } \\
\text { poles }\end{array}$ & $\begin{array}{c}\text { Other } \\
\text { poles }\end{array}$ & TP & FN & FP & Completeness & Correctness & Quality \\
$\mathbf{0 . 3}$ & 14 & 15 & 6 & 8 & 0 & 0.43 & 1.00 & 0.43 \\
$\mathbf{0 . 4}$ & 14 & 15 & 8 & 6 & 0 & 0.57 & 1.00 & 0.57 \\
$\mathbf{0 . 5}$ & 14 & 15 & 9 & 5 & 1 & 0.64 & 0.90 & 0.60 \\
$\mathbf{0 . 6}$ & 14 & 15 & 11 & 3 & 1 & 0.79 & 0.92 & 0.73
\end{tabular}

Table 2. Evaluation results.

\section{REFERENCES}

Awrangjeb, M. and C. S. Fraser (2014). "Automatic Segmentation of Raw LIDAR Data for Extraction of Building Roofs." Remote Sensing 6(5): 3716-3751.

Brogan, M., et al. (2013). "Assessment of stereo camera calibration techniques for a portable mobile mapping system." IET Computer Vision 7(3): 209-217.

Cabo, C., et al. (2014). "An algorithm for automatic detection of pole-like street furniture objects from Mobile Laser Scanner point clouds." ISPRS Journal of Photogrammetry and Remote Sensing 87: 47-56.

Chen, Y.-Z., et al. (2007). Amobile System Combining Laser Scanners and Cameras for Urban Spatial Objects Extraction 2007 International Conference on Machine Learning and Cybernetics, IEEE.

El-Halawany, S. I. and D. D. Lichti (2011). Detection of road poles from mobile terrestrial laser scanner point cloud. MultiPlatform/Multi-Sensor Remote Sensing and Mapping (M2RSM), 2011 International Workshop on, IEEE.

El-Halawany, S. I. and D. D. Lichti (2013). "Detecting road poles from mobile terrestrial laser scanning data." GIScience \& Remote Sensing 50(6): 704-722.

Fleischer, K. and H.-H. Nagel (2001). Machine-vision-based detection and tracking of stationary infrastructural objects beside inner-city roads. Intelligent Transportation Systems, 2001. Proceedings. 2001 IEEE, IEEE.

Gong, J., et al. (2012). Mobile terrestrial laser scanning for highway inventory data collection. Proceedings of International Conference on Computing in Civil Engineering, Clearwater Beach, FL, USA.
Guan, H., et al. (2015). "Iterative tensor voting for pavement crack extraction using mobile laser scanning data." IEEE Transactions on Geoscience and Remote Sensing 53(3): 15271537.

Guan, H., et al. (2015). "Deep learning-based tree classification using mobile LiDAR data." Remote Sensing Letters 6(11): 864873.

Hu, Y., et al. (2011). A novel approach to extracting street lamps from vehicle-borne laser data. Geoinformatics, 2011 19th International Conference on, IEEE.

Kukko, A., et al. (2009). Mobile mapping system and computing methods for modelling of road environment. 2009 Joint Urban Remote Sensing Event, IEEE.

Lehtomäki, M., et al. (2010). "Detection of vertical pole-like objects in a road environment using vehicle-based laser scanning data." Remote Sensing 2(3): 641-664.

Murray, S., et al. (2011). "Mobile mapping system for the automated detection and analysis of road delineation." IET intelligent transport systems 5(4): 221-230.

Pingel, T. J., et al. (2013). "An improved simple morphological filter for the terrain classification of airborne LIDAR data." ISPRS Journal of Photogrammetry and Remote Sensing 77: 21 30.

$\mathrm{Pu}, \mathrm{S}$., et al. (2011). "Recognizing basic structures from mobile laser scanning data for road inventory studies." ISPRS Journal of Photogrammetry and Remote Sensing 66(6): S28-S39.

Rutzinger, M., et al. (2009). "A comparison of evaluation techniques for building extraction from airborne laser scanning." Selected Topics in Applied Earth Observations and Remote Sensing, IEEE Journal of 2(1): 11-20

Tran, T. N., et al. (2013). "Revised DBSCAN algorithm to cluster data with dense adjacent clusters." Chemometrics and Intelligent Laboratory Systems 120: 92-96. 
Yokoyama, H., et al. (2011). "Pole-like objects recognition from mobile laser scanning data using smoothing and principal component analysis." International Archives of Photogrammetry, Remote Sensing and Spatial Information Sciences 38(5/W12): 115-120.

Yokoyama, H., et al. (2013). "Detection and classification of pole-like objects from mobile laser scanning data of urban environments." International Journal of CAD/CAM 13(2).

Zai, D., et al. (2015). Inventory of 3D street lighting poles using mobile laser scanning point clouds. Geoscience and Remote Sensing Symposium (IGARSS), 2015 IEEE International, IEEE. 